\title{
I. Theorie
}

\section{Hans-Joachim Hoffmann-Nowotny}

\section{Soziologische Marginalien zur Marginalisierung durch „illegitime" Geburt}

I.

Aus soziologischer Sicht ist es ein nicht unbedingt überzeugender Sprachgebrauch, außerehelich geborene Kinder als „illegitim“ zu bezeichnen, wird doch das Konzept der "Legitimität" (insbesondere im Anschluß an Max Weber) bzw. sein Gegenteil, "Illegitimität", durchwegs im Zusammenhang mit der Frage nach der "Geltung" einer „Ordnung“ verwendet. Danach ist primär eine „Ordnung“ legitim oder illegitim, nicht aber ein Handeln oder gar ein Erdulden, wie die Tatsache der Geburt unter dem Aspekt des Status der Beziehung der Eltern. Dieses semantische Problem kann hier nicht weiter verfolgt werden, sondern ist wohl nur historisch zu erhellen.

„Handeln“ oder eine „soziale Beziehung“ können allerdings - wie Weber sagt - „von seiten der Beteiligten an der Vorstellung vom Bestehen einer legitimen Ordnung orientiert werden"'. Auf diese Weise wird eine Verbindung zwischen "Ordnung" und „sozialer Beziehung" hergestellt, so daß im hier interessierenden Zusammenhang, also wenn man Geburten danach klassifiziert, ob die Beziehung der Eltern eines Kindes an einer Legitimität beanspruchenden „Ordnung“ orientiert war oder nicht, die „illegitime“ Geburt dennoch sinnvoll in den Rahmen der eben skizzierten Begrifflichkeit eingepaßt werden kann.

\section{II.}

Nach dem eingangs Gesagten kann „Illegitimität“ erst dann auftreten und als solche etikettiert werden, wenn eine bestimmte Ordnung eingeführt worden ist und mit Geltungsanspruch auftritt. Vor diesem Hintergrund kann "Illegitimität" entweder erworben sein oder zugeschrieben werden; anders formuliert, sie hat eine aktive und eine passive Komponente. Die aktive Komponente liegt dann vor, wenn ein bestimmtes Handeln gegen eine „Ordnung“ verstößt, Illegitimität also von dem oder den Handelnden erwor-

1 Max Weber, Wirtschaft und Gesellschaft. Grundriß der verstehenden Soziologie. Hrsg. v. Johannes Winckelmann (Köln/Berlin 1964) 22; im folgenden zitiert: Weber, Wirtschaft. 
ben wird. Die passive Komponente ist gegeben, wenn Illegitimität ohne eigenes Zutun zugeschrieben wird: Das illegitime Kind ist hier das treffendste Beispiel.

"Ordnungen“ im weitesten Sinne sind Teil der Kultur-Dimension der Gesellschaft. Die zweite zentrale Dimension von Gesellschaft kann mit dem Begriff der Struktur bezeichnet werden. In einer Kurzdefinition bezeichnet Kultur das legitimierende Symbolsystem, Struktur das machthaltige Positionssystem einer Gesellschaft. ${ }^{2}$ Nach einer von mir entwickelten soziologischen Theorie - dem Struktur/Kultur-Paradigma - sind beide Dimensionen wechselseitig miteinander verbunden (struktureller Wandel zieht kulturellen Wandel nach sich und vice versa) und jede Dimension kann zudem Eigendynamik entwickeln (ein strukturell einmal angestoßener kultureller Wandel kann sich verselbständigen und vice versa). Struktur und Kultur eines sozietalen Systems können einander entsprechen. Dann befindet sich ein solches System im Gleichgewicht und kann als "stabil" bezeichnet werden. Durch endogene oder exogene Anstöße kann ein Gleichgewicht gestört werden. Dann entsprechen das Symbolsystem und das Positionssystem einander nicht mehr, woraus Spannungen resultieren, die auf eine neue Entsprechung zielen, d.h. soziokulturellen Wandel in Gang setzen.

Das Phänomen „Illegitimität" darf nun - obwohl dies nahezuliegen scheint - nicht nur auf der normativen Ebene der kanonischen "Ordnung“, der Ebene der Kultur also, behandelt werden; es hat vielmehr immer auch ein strukturelles Pendant. Es hat ein strukturelles Pendant in seiner Entstehung, also wenn mit Definitionsmacht ausgestattete Institutionen - z.B. die Kirche - bzw. Positionsinhaber - z.B. der Papst - definieren, was als "legitim“ gelten soll und was folglich als „illegitim“ bezeichnet werden kann. Das Phänomen „Illegitimität" hat aber auch eine strukturelle Komponente in seinen Folgen: Abweichung von kulturell gebotenen Ordnungen zieht in aller Regel strukturelle Marginalität nach sich; ausgedrückt z.B. im Verlust von Positionen (sozialer Abstieg) oder der Sperrung des Zugangs dazu, z.B. als Zugangssperre zu den höheren Weihen oder als Ausschluß vom Erbgang. ${ }^{3}$ Man darf annehmen, daß mit Definitionsmacht ausgestattete Institutionen sich bei der Ordnungssetzung nicht zuletzt von strukturell begründeten Motiven, nämlich dem Bestreben der Machterhaltung oder der Machtausweitung leiten lassen,

2 Die Werte, Normen oder auch Ideologien des Kulturbereichs können eine bestimmte Strukrur legitimieren, während strukturelle Macht ihrerseits zur Erhaltung von Werten, Normen und Ideologien eingesetzt werden kann. Ein komplexes Modell soziokulturellen Wandels findet sich in Hans-Joachim HoffmannNowotny, Soziologische Notizen zu einigen Problemen des Wertwandels, in: Wertwandel und gesellschaftlicher Wandel. Hrsg. v. Helmut Klages, Peter Kmieciak (Frankfurt/New York 3. Aufl. 1984) 61-66.

3 An dieser Stelle ist darauf hinzuweisen, daß es natürlich nicht nur eine "Ordnung" gibt, sondern - entsprechend dem Differenzierungsgrad einer Gesellschaft - immer mehrere Ordnungen existieren, die einander nebengeordnet sind bzw. zueinander im Verhältnis von Über- oder Unterordnung stehen. So ist es denn auch naheliegend zu erwarten, daß die Kirche zwar in ihrem unmittelbaren Machtbereich illegitime Kinder marginalisieren konnte, daß aber z.B. der Adel sich relativ frei darin fühlen konnte zu bestimmen, ob und in welchem Maße illegitime Kinder gegenüber legitimen benachteiligt wurden. Ähnliches gilt auch für die Zünfte, für die es durchaus nicht selbstverständlich sein mußte, illegitim Geborene als Mitglieder oder als Lehrlinge abzuweisen. Schließlich darf auch vermutet werden, daß hochrangige Mitglieder des Klerus ihre strukturelle Macht dazu einsetzen konnten, ihre Kinder besser vor der Marginalisierung zu schützen, als dies niederrangigen Mitgliedern möglich war. 
die dann ihrerseits kulturell legitimiert werden müssen, um kein Ungleichgewicht zwischen der strukturellen und der kulturellen Dimension entstehen zu lassen.

\section{III.}

Im Falle der Kirche liegt es nahe zu vermuten, daß die Erlangung der Jurisdiktion über und des Monopols für die Eheschließung dem Motiv der Machtausweitung entsprang, einer Machtausweitung, die - und dies verdient festgehalten zu werden - sogar sakramental legitimiert wurde. Wenn diese Jurisdiktion und das ihr entsprechende Monopol durchgesetzt waren, hatte die Kirche einen der zentralsten Bereiche menschlicher Existenz ihrer Herrschaft unterworfen. ${ }^{4}$ Auch die Einführung des Zölibats kann aus dieser Perspektive gesehen werden: Zum einen stärkte er die Bindung der Geistlichen an die Kirche, indem Eheschließung und die Gründung einer Familie untersagt wurden, zum anderen blieb die Amts- und Statussukzession - zumindest idealiter - in der Entscheidungsgewalt der Institution.

Wie wir wissen, konnte die katholische Kirche den Alleinanspruch in bezug auf die Eheschließung keineswegs von einem Tag auf den anderen, sondern erst im Verlaufe eines vom 12. bis in das 16. Jahrhundert gehenden Prozesses sukzessive de iure (wenn auch nicht immer de facto) durchsetzen (vollumfänglich etabliert wurde dieser Anspruch wohl erst nach der Reformation), ehe sie ihn dann Jahrhunderte später zunächst mit dem Staat teilen und schließlich an ihn abtreten mußte.

Die normative Regelung der Ehe - die wie alle Regelungen aus vielerlei Gründen in der Praxis nicht universell durchsetzbar war - zeitigte nun aber die Konsequenz, daß Kinder aus einer nicht kirchlich sanktionierten Verbindung „illegitim“ wurden. Damit hätte nicht - jedenfalls nicht notwendigerweise - deren Marginalisierung verbunden sein müssen, wie sie z.B. im besagten Verbot des Zugangs zur Priesterschaft oder in der Einschränkung des Erbrechtes zum Ausdruck kam. Dies gilt um so mehr, als solche Kinder - ich erinnere an das oben Gesagte - den Status der Illegitimität ja nicht durch eigenes Handeln erworben hatten.

Man hätte sich auch eine ausschließliche Sanktionierung der Eltern, die in den reformierten Gebieten dann zum Teil besonders rigoros gehandhabt wurde, vorstellen können. So berichtet Bickel für das 18. Jahrhundert, „daß die geringe Zahl unehelicher Geburten, die sich in den Taufregistern findet, ohne Zweifel darauf zurückzuführen ist, daß die Erzeugung außerehelicher Kinder streng bestraft und daher nach Möglichkeit verheim-

4 Mit dieser Argumentation sollen andere Motive der Kirche - z.B. solche kultureller Art - nicht ausgeschlossen werden. So hat ja bekanntlich Calvin versucht, die von ihm mit besonderer Strenge postulierte Monogamie aus der Bibel abzuleiten, auch wenn dies recht konstruiert erscheint (vgl. dazu Gudrun Beckmann, Die christliche Ehe. Der Einfluß Calvins auf die moderne Ehe, in: System Familie 4 [1991] 26-37; im folgenden zitiert: Beckmann, Ehe). Luther hielt zwar - im Gegensatz zur Auffassung der katholischen Kirche - die Ehe für ein „eußerlich leyplich ding wie andere hantrierung", argumentierte aber insofern strukturell, als er meint, die Ehe sei die Grundlage des menschlichen Gemeinwesens, das „zergehen und zerfallen müßte, wo kein ordentlicher und gewisser Stand der Ehe wäre" (Zitat ebd., 27). 
licht wurde." In Basel mußten 1770 Hebammen „bei ihrem Eid“ ermahnt werden, uneheliche Kinder anzuzeigen, "dieweilen“ - wie es hieß - „das abscheuliche Laster des Kindermordes bei diesen verderbten Zeiten fast gemein wird." 6 Wie wir sehen, wurden aber auch über diesen Sanktionsmechanismus in letzter Konsequenz nicht die Eltern, sondern die Kinder marginalisiert, in diesem Falle sogar in nicht mehr rückgängig zu machender Weise. Man darf gewiß unterstellen, daß der Kindermord eine nicht-intendierte Nebenfolge der Institutionalisierung der Eheschließung oder auch des Zölibats war. In bezug auf die Zuschreibung des Status der Illegitimät gilt das Gleiche mit Sicherheit nicht, wurde er doch explizit definiert und mit negativen Sanktionen belegt.

Darf man - als Frage formuliert - vermuten, daß damit ein „Ehezwang“ ausgeübt werden sollte? Daß potentielle Eltern - also jedenfalls diejenigen, die für eine EheschlieBung überhaupt zugelassen waren und damit dafür in Frage kamen - sich dem kirchlichen Ehemonopol fügen sollten, um Nachteile für ihre Kinder zu vermeiden? Daß es sich dabei um ganz erhebliche Nachteile handelte, muß nicht besonders betont werden. Die Kirche des Mittelalters war nicht nur der porenteste Arbeitgeber dieser Epoche, sondern sie bot darüber hinaus auch - allenfalls noch mit dem allerdings im wahrsten Sinne des Wortes lebensgefährlichen Kriegsdienst vergleichbar - praktisch als einzige Institution in einer ständisch geschlossenen Gesellschaft die Chance sozialer Aufwärtsmobilität. Arbeitsmöglichkeiten - gesicherte Arbeit dazu in meist unsicheren Zeiten - wie Aufstiegschancen zu entziehen, bedeutete nicht gerade wenig. Der starken Definitionsmacht entsprach eine starke Sanktionsmacht: was kulturell gefordert wurde, konnte strukturell entweder auch durchgesetzt oder zumindest mit massiven Sanktionen belegt werden.

Mit dem Ehegebor für die Laien „entstanden“ mindestens drei Kategorien illegitimer Kinder:

1) Kinder von prinzipiell zur Ehe zugelassenen, aber außerhalb des Ehestandes lebenden Personen,

2) Kinder von Personen im Ehestand, die außerehelich gezeugt waren, und schließlich

3) Kinder von nicht zur Ehe zugelassenen Personen.

In bezug auf die letzte Gruppe ist zwar anzumerken, daß die Kirche im Prinzip für Laien kein Eheverbor kannte, daß jedoch geistliche Grundherren wie Bischöfe oder Klöster ihren Hörigen durchaus die Eheschließung oft in gleicher Weise untersagten (oder zumindest erschwerten) wie weltliche Grundherren. Klöster schlossen - wenn ihnen die Heiratsmöglichkeiten zu stark beschränkt erschienen - „unter sich Verträge ab, die ihren Hörigen die Ehe mit Eigenleuten aller Vertragschließenden gestatteten. Die Kinder wurden geteilt"?.

Mit dem 1139 eingeführten Eheverbor für Weltpriester (und dem für monastische Lebensformen in den jeweiligen Ordensregeln schon lange vorher festgeschriebenen Zölibat) war eine vierte Gruppe von illegitimen Kindern programmiert, nämlich Kinder weltlicher Priester (bzw. Kinder von Mönchen oder Nonnen). Daß deren Eltern - beschrän-

5 Walter Bickel, Bevölkerungsgeschichte und Bevölkerungspolitik der Schweiz seit dem Ausgang des Mittelalters (Zürich 1947) 78. Im folgenden zitiert: Bickeh, Bevölkerungsgeschichte.

6 Ebd., 78.

7 Ebd., 47. 
ken wir uns hier auf die priesterlichen Väter - ein besonderes Interesse daran haben mußten, die mit der Illegitimität verbundenen negativen Konsequenzen von ihren Kindern abzuwenden, ist unmittelbar evident und auch leicht nachfühlbar. Wußten diese Väter doch aus eigener Erfahrung - und damit besser als alle anderen - um die Bedeutung der Kirche als Arbeitgeber wie als Aufstiegskanal.

Wiederum kann ich als historisch dilettierender Soziologe nur fragen, ob beim Prozeß der Institutionalisierung von Ehe und Zölibat die damit einhergehende Illegitimisierung von Priesterkindern wissentlich als unvermeidliches Problem in Kauf genommen wurde oder ob dieses Problem als nicht-intendierte Nebenfolge auftrat. Wie dem auch sei, man darf annehmen, daß sich im einen wie im anderen Falle Regelungen aufdrängten, welche die Konsequenzen dieser Zuschreibung in Grenzen hielten - wenn der Sachverhalt der Illegitimät von Priesterkindern schon nicht zu vermeiden war. Als Instrument zur Behebung der aus dem Illegitimitätsstatus resultierenden Marginalisierung stand die Dispens zur Verfügung. Es ist denn auch wahrscheinlich kein Zufall, daß die Dispenspraxis gegen Ende des 12. Jahrhunderts einsetzte; also bald nach der Einführung des Zölibats, während die kanonischen Grundlagen der Eheschließung - nach einer viele Jahrhunderte währenden Auseinandersetzung darüber ${ }^{8}$ - erst im frühen 13. Jahrhundert gelegt wurden. In der Folge wurde die Dispens, die Ende des 13. Jahrhunderts volle Praxis geworden war, offenbar großzügig gehandhabt.

Warum "drängte" sich aber - wie ich formuliert habe - eine Regelung wie die Dispens auf? Man darf vielleicht unterstellen, daß zum einen angesichts der anscheinenden Massenhaftigkeit der Erzeugung von Priesterkindern der Kirche bewußt war, daß sie hier an die Grenzen der strukturellen Durchsetzung kultureller Ordnungen gestoßen war, ohne daß sie deshalb aber auf den Durchsetzungsanspruch verzichten wollte. Zum anderen ist es vermutlich nicht abwegig anzunehmen, daß auch die Kirchenoberen selbst, die ja in der hier diskutierten Hinsicht ebenfalls nicht ohne Fehl und Tadel waren, zusätzlich ein unmittelbares persönliches Interesse hatten, Schaden von ihren Kindern abzuwenden. Institutionelles und persönliches Interesse der kirchlichen Eliten hätten so bei der Etablierung der Dispense zusammengewirkt.

Die Vermeidung der Marginalisierung hätte - wie schon gesagt - natürlich auch ein Verzicht von vornherein auf die Zuschreibung von Illegitimität bewirkt, was die Dispens also überflüssig gemacht hätte. Auch humanitäre Überlegungen hätten nach dem Prinzip „keine Strafe ohne Schuld“ ein solches Vorgehen nahelegen können. Dies ist allerdings römisch-rechtlich und aufklärerisch inspiriertes Denken, das der Kultur einer Institution fernliegen mußte, die in Gestalt der Erbsünde Schuld ohne eigenes Verschulden ad infinitum weitergegeben sah.

Wiederum hat man sich aber wohl auch eine strukturelle Komponente als wirksam vorzustellen. Die Dispens ist ein Machtinstrument; der Verzicht darauf, mittels der Dispense selbst geschaffene Marginalität wieder aufheben zu können, hätte einen Machtverzicht bedeutet. Es gab keinen Grund, dies ohne Not zu tun. Parallelen zu diesem Vorgang lassen sich in vielfacher Form als Varianten des gleichen Grundmusters in der 
Geschichte der Kirche zuhauf finden. Im ego te absolvo erfährt der praktizierende Katholik noch heute eine spezielle Schlußformel des zentralsten Musters.

\section{IV.}

Nun möchte ich mich vom Mittelalter in die Neuzeit und in die neueste Zeit begeben.? Wie schon gesagt, hatten die Reformatoren die Sanktionierung von Illegitimität eher noch verschärft, nicht zuletzt dadurch, daß es jetzt keine Dispens mehr gab. Illegitimität und bestimmte ihrer Folgen (z.B. im Namens- und Erbrecht) wurden schließlich aber auch in das staatlich bürgerliche Recht übernommen.

Mit dem Aufkommen der die Fesseln der ständischen Feudalgesellschaft sprengenden "bürgerlichen Gesellschaft" entwickelte sich - im wesentlichen auf der Basis der protestantischen Ethik Calvins - seit dem Ende des 18. Jahrhunderts die „bürgerliche“ Ehe und Familie. ${ }^{10}$ Zunächst auf die „Bürger“ im engeren Sinne des Wortes beschränkt, wurde sie seit etwa der letzten Jahrhundertwende (bei nicht zu vernachlässigenden schichtspezifischen Unterschieden) zu einer in allen sozialen Schichten dominierenden Form des Zusammenlebens im Primärbereich und, als schließlich weitgehend vom Staat kontrollierte Institution, zu einer Art von Idealtyp. ${ }^{11}$ Als "the golden age of marriage ${ }^{\text {"12 }}$ ist aber erst die Zeit nach dem Zweiten Weltkrieg bis Mitte der sechziger Jahre dieses Jahrhunderts zu bezeichnen - eine Tatsache, die außerhalb der Demographie noch kaum bekannt ist. In unseren Breiten gehen in dieser Zeit mehr als $90 \%$ aller Männer und Frauen eine auch formell geschlossene eheliche Beziehung ein, und entsprechend gering wird die Zahl außerehelicher Kinder. Im Jahre 1965 beträgt deren Anteil an allen Geburten in Italien $2.0 \%$, in der Schweiz 3.9\%, in der Bundesrepublik Deutschland $4.7 \%$ und in Frankreich $5.9 \%$. Allerdings werden sie zunächst immer noch weitgehend als "illegitime“ Kinder bezeichnet, so z.B. selbst in einem Standardwerk der Familiensoziologie, René Königs 1974 erschienener Schrift „Die Familie der Gegenwart“. ${ }^{13}$

Die die Feudalgesellschaft ablösende „bürgerliche Gesellschaft“ hat sich ihrerseits permanent gewandelt, und als Folge dessen gab es auch vielfältige Veränderungen im Bereich

9 Ein Beispiel für einen in der Neuzeit von weltlicher Macht drastisch ausgeübten Ehezwang - der gleichzeitig die Illegitimität eines Kindes verhinderte - findet sich im Heiratsbuch von Müllheim (Baden): „1737 den 6. Novembris ist Johannes Meyer von Mengen auff Serenissimi hohen Befehl in der Kirchen allhier von dem Herrn Diacono Zanden mit Barbara Pfisterin, welche Meyer sub promissione matrimonii geschwängert, copulirt worden, und weil ersagter Meyer die Pfisterin absolute nicht hat heurathen wollen, ist er von 4 Wächtern armata manu in die Kirchen geführt, zum Altar hingeschleppt, seine Hand mit Gewalt in die Hand der Pfisterin eingeschlagen worden, und da er beständig ,Nein“ sagte: ,Ich will sie nicht', etc. hat Herr Diaconus ex mandato Serenissimi ,Ja' gesagt." Wilhelm Ebel, Curiosa iuris germanici (Götringen 1968) 71.

10 Edward Shorter, The Making of the Modern Family (New York 1975); Michael Mitterauer, Reinhard Sieder, Vom Patriarchen zur Partnerschaft. Zum Strukturwandel der Familie (München 1977); Heinz Reif (Hrsg.), Die Familie in der Geschichte (Göttingen 1982).

11 Brigitte Berger, Peter L. Berger, The War over the Family. Capturing the Middle Ground (Garden City N.Y. 1983).

12 Patrick Festy, On the New Context of Marriage in Western Europe, in: Population and Development Rev. 6.2 (1980) 311-315.

13 René König. Die Familie der Gegenwart. Ein interkultureller Vergleich (München 1974). 
der Beziehungen der Geschlechter. In jüngster Zeit erfahren Ehe und Familie - und allgemeiner: Formen des menschlichen Lebens im Primärbereich - aber einen Wandel, der sich anscheinend gegenüber den säkularen Veränderungen in diesem Bereich erheblich beschleunigt hat. ${ }^{14}$ Einige wenige Hinweise sollen diesen Wandel verdeutlichen:

Bei einer Betrachtung der Entwicklung des Anteils der „vollständigen“ Familien an allen Haushalten zeigt sich über die letzten 20 Jahre eine permanente Abnahme. ${ }^{15}$ In vielen europäischen Ländern ist dieser Anteil inzwischen unter $40 \%$ gesunken. In Großstädten wie Zürich macht er nur noch $20 \%$ aus. Der klassische Familienhaushalt hat damit definitiv einen „Minderheitsstatus“ erlangt. Der Index der Gesamtheiratshäufigkeit, der Auskunft darüber gibt, wie groß der Anteil derjenigen ist, die bis zum Alter von unter 50 Jahren eine erste Ehe eingehen, läßt - Konstanz der gegenwärtigen Verhältnisse vorausgesetzt - erkennen, daß dann in der Schweiz 69\%, in der Bundesrepublik rund $58 \%$, in Frankreich 52\% und in Schweden nur 50\% aller Männer heiraten würden. Die Ehe verliert als Lebensform an Bedeutung; die Gründung von Familien nimmt ab, und die Familien, die gegründet werden, werden immer kleiner. Die Zahl der Kinder, die heute ja im wesentlichen die Größe eines Familienhaushaltes bestimmt, beträgt in der Bundesrepublik 1.5 pro Frau und hat inzwischen in Italien den historischen Tiefstwert von 1.3 Kindern pro Frau erreichr. Ehen und Familien lösen sich früher und zunehmend häufiger auf, auch wenn Familien etwas weniger früh und etwas weniger schnell aufgelöst werden als kinderlose Ehen. ${ }^{16}$ Ehescheidungen nehmen kontinuierlich zu. In Deutschland wird nach dem heutigen Stand rund ein Drittel aller Ehen geschieden, mit entsprechenden Konsequenzen für die Zahl und Zusammensetzung der Haushalte. Die ehemalige Sowjetunion nähert sich der $40 \%$-Grenze, in Schweden werden $44 \%$ und in den USA etwa $50 \%$ aller Ehen geschieden. Ein-Eltern-Familien nehmen mit hohen Wachstumsraten zu. Wiederverheiratungen werden seltener und sind noch mehr durch Scheidung gefährdet als Erst-Ehen. Kohabitationen - d.h. nicht-eheliche Formen des Zusammenlebens - sind in Zunahme begriffen und ersetzen Ehen - aber noch nicht immer Familien - und zeichnen sich zudem durch ein hohes $\mathrm{Maß}$ an Instabilität aus. Und als Single zu leben, scheint schließlich mehr als eine Mode zu sein.

Dieser Wandel, wie auch seine Beschleunigung, gilt einmal für alle entwickelten Gesellschaften, und zwar offenbar unabhängig von deren ideologischer Ausrichtung oder

14 Kurt Lüscher, Franz Schultheis, Michael Wehrspaun (Hrsg.), Die „postmoderne“ Familie. Familiale Stategien und Familienpolitik in einer Übergangszeit (Konstanz 1988); Katja Boh u.a., Changing Patterns of European Family Life. A Comparative Analysis of 14 European Countries (London/New York 1989); im folgenden zitiert: Boh, Patterns; Statistisches Bundesamt, Familien heute. Strukturen, Verläufe und Einstellungen (Stuttgart 1990); Hans-Joachim Hoffmann-Nowotny, Weibliche Erwerbstätigkeit und Kinderzahl, in: Frauensituation. Veränderungen in den letzten zwanzig Jahren. Hrsg. v. Uta Gerhardt, Yvonne Schütze (Frankfurt a.M. 1988); im folgenden zitiert: Hoffmann-Nowotny, Erwerbstätigkeit; Alain Monnier, Catherine Guibert-Lantoine, La conjoncture démographique. L'Europe et les pays développés d'Outre-Mer, in: Population 46.4 (1991) 941-964.

15 Louis Roussel, L'évolution récente de la structure des ménages dans quelques pays industriels, in: Population 42.6 (1986) 913-934.

${ }_{16}$ Jürg Willi, Was hält Paare zusammen? Der Prozeß des Zusammenlebens in psychoökologischer Sicht (Hamburg 1991). 
politischem Regime - wie ein Ost-West-Vergleich unschwer erkennen läßt ${ }^{17}$ - und läßt sich auch schon in den weniger entwickelten Ländern konstatieren. Zum anderen erweckt er den Anschein ${ }^{18}$, daß es sich nicht mehr um Veränderungen handelt, bei denen die Grundmuster im Prinzip gleich bleiben, sondern daß unsere Lebensformen dabei sind, recht grundlegend anders zu werden. $\mathrm{Ob}$ man diese Entwicklung als einen revolutionären Bruch mit der Vergangenheit bezeichnen kann ${ }^{19}$, oder ob man von besonders schnellen evolutionären Veränderungen sprechen soll ${ }^{20}$, ist hingegen eine offene Frage, welche die Historiker eines ferneren Tages beantworten werden.

Hinsichtlich unseres Themas fällt bei diesem Wandel und mit Blick auf seine Fortsetzung insbesondere ins Gewicht, daß es vermutlich wenig Grund gibt anzunehmen, Ehe und Familie in ihrer traditionellen Gestalt könnten und würden als "mainstream"Modelle für zukünftige Lebensmuster überleben. Ich habe dies in einigen Arbeiten ausführlich erläutert und als Konsequenz gesamtgesellschaftlichen strukturellen und kulturellen Wandels erklärt. ${ }^{21}$

Mit Bezug auf den angesprochenen Wandel ist es nun weniger bedeutsam, daß Ehe und Familie weiterhin ais rechtliche Institutionen existieren, so daß rein formal weiterhin nach "legitimen“ und „illegitimen“ Kindern unterschieden werden kann. Wichtiger ist vielmehr, daß Ehe und Familie sich als sozial verbindliche Institutionen auflösen, d.h. als internalisierte, sozial geteilte und kontrollierte Muster von Rollen und Normen einer bestimmten ("legitimen") Ordnung des Zusammenlebens. Es ist zwar weiterhin „legitim" zu heiraten und damit "legitime“ Kinder zu zeugen. Es liegt nun aber insofern eine kulturelle Asymmetrie vor, als es gleichzeitig nicht mehr „illegitim“ ist, ohne staatlichen und/oder kirchlichen Segen zusammenzuleben und Kinder zu haben, die folglich auch nicht „illegitim“ sein können. Ja selbst der Realisierung des Wunsches einer Frau, ein Kind haben zu wollen, eine Partnerschaft aber ausdrücklich abzulehnen, haftet kaum noch ein Makel an.

Gerade weil aber institutionelle Restriktionen reduziert wurden oder sogar völlig verschwanden (zusammen mit existenziellen Abhängigkeiten) sind Primärgruppen zu einem "Reich der Freiheit" geworden, in dem man unter einem System "gesellschaftlicher" Werte $^{22}$ (persönliche Autonomie, Selbstverwirklichung, Emanzipation etc.), die zentraler

17 Boh, Patterns (wie Anm. 14); Barbara Bertram, Walter Friedrich, Otmar Kabat vel Job, Adam und Eva heute (Leipzig 1988).

18 Hans-Joachim Hoffmann-Noworny, Lebensformen und Lebensstile der (Post-)Moderne, in: Familiendynamik, $16 / 4$ (1991) 299-321; im folgenden zitiert: Hoffmann-Nowotny, Lebensformen.

19 Dirk J. van de Kaa, Emerging Issues in Demographic Research for Contemporary Europe. Paper presented at the Conference of Population Research in Great Britain (University of East Anglia 1986).

20 Robert L. Cliquet, De Tweede Demografische Transitie. Feit of fictie?, in: Bevolking en Gezin 2 (1991) $1-42$.

21 Hans-Joachim Hoffmann-Nowotny, Auf dem Wege zur autistischen Gesellschaft?, in: Eheschließung und Familienbildung heute. Hrsg. v. Sabine Rupp, Karl Schwarz, Max Wingen (Wiesbaden 1980) 161-185; ders., The Future of the Family, in: Plenaries of the European Population Conference (Helsinki 1987) 113200; ders., Lebensformen (wie Anm. 18).

22 "Gesellschaftlich" wird hier verstanden in Anlehnung an das von Ferdinand Tönnies geprägte Gegensatzpaar "Gemeinschaft" und "Gesellschaft". Vgl. Ferdinand Tönnies, Gemeinschaft und Gesellschaft. Grundbegriffe der reinen Soziologie (1887, ND Darmstadt 1979). 
Teil der Ideologie des Individualismus sind, zusammenleben kann, wenn auch in fragiler Weise. Die Formel der „anarchistischen Erkenntnistheorie ${ }^{\text {«23 }}$ Paul Feyerabends, „anything goes“, ist auch auf die Gestaltung von Lebensformen anwendbar.

Es erscheint fast paradox, daß die De-Institutionalisierung und Individualisierung zum einen die andauernde Attraktivität nicht-institutionalisierter Lebensformen ausmachen, zum anderen aber gleichzeitig Ursache ihrer Fragilität sind. Dies wird sich voraussichtlich in der absehbaren Zukunft kaum ändern. Es ist jedoch nicht unwahrscheinlich, daß mit weiter abnehmender Institutionalisierung auf der einen und zunehmender Individualisierung auf der anderen Seite ein Leben als "Single“ eine zunehmend attraktive Alternative zur Gründung einer dauerhaften Primärgruppe wird. Daß dies strukturell und kulturell nicht nur ermöglicht, sondern sogar nahegelegt wird, haben ich an anderer Stelle hinreichend begründet. ${ }^{24}$

Mit Blick auf die Zukunft kollektiver Lebensformen halte ich es für höchst unwahrscheinlich, daß es in absehbarer Zeit noch einmal ein Muster des Zusammenlebens geben wird, das so dominant und langlebig sein könnte wie das der kirchlichen oder der bürgerlichen Ehe und Familie. Die Auflösung strukturell und kulturell verankerter (d.h. gesellschaftlich vorgegebener und verpflichtender) Modelle des Zusammenlebens im Primärbereich und die mutmaßliche Unwahrscheinlichkeit ihrer Wiederherstellung machen es vielmehr unabdingbar, von einer großen Varietät permanent wechselnder und nicht sehr stabiler Lebensformen auszugehen, die mehr oder weniger an "gesellschaftliche“ Bedingungen und deren Wandel angepaßt sind und die dauerhafte gesamtgesellschaftliche Anomie zum Ausdruck bringen. Angesichts des Fehlens eines allgemein verbindlichen Musters, wie die Quasi-Monopolinstitution der „bürgerlichen“ Ehe und Familie es darstellte, ist eine Vielzahl von Versuchen zu erwarten, in denen nach dem "trial and error"Prinzip individuell bestimmte Lebensformen eingegangen, erprobt und häufig auch wieder schnell aufgegeben werden. ${ }^{25}$

Die Zeit des von der Kirche eingeführten und durchgesetzten sowie schließlich vom Staat übernommenen Monopols der Sanktionierung von Lebensformen neigt sich also offenbar ihrem Ende zu. Die Zahl der Eheschließungen hat in den letzten 25 Jahren zum Teil dramatisch abgenommen. Da aber der Kinderwunsch, auch wenn er sich in der gleichen Zeitspanne stark verringert hat ${ }^{26}$, nicht gänzlich verschwunden ist, nehmen auch Zahl und Anteil von - im alten Sprachgebrauch - „illegitimen“ Kindern stark zu. 1989/90 macht der Anteil außerehelicher Kinder an allen Geburten in der Schweiz 6.1\% aus, in Italien 6.3\%, in der Bundesrepublik 10.2\%, in Frankreich 28.2\% (in der „alten“ DDR 33.6\%) und beträgt in Schweden $51.8 \%$.

Dieser strukturelle Wandel hat auch einen terminologischen (kulturellen) Wandel zur Folge: Aus „illegitimen“ Kindern wurden „uneheliche“ und aus diesen „außereheliche“

23 Paul Feyerabend, Wider den Methodenzwang. Skizze einer anarchistischen Erkenntnistheorie (Frankfurt a.M. 1976).

24 Hoffmann-Nowotny, Lebensformen (wie Anm. 18).

25 Britta Hoem, Jan M. Hoem, Dissolution in Sweden. The Break-Up of Conjugal Unions to Swedish Women Born in 1936-60. Stockholm Research Reports, in: Demography 45 (Stockholm 1985).

26 Hoffmann-Nowotny, Erwerbstätigkeit (wie Anm. 14). 
Kinder; „extra-marital births" ist heute der international gebräuchliche Standardbegriff. Es ist aber abzusehen, daß die Kategorisierung einer Geburt nach der Form der Beziehung von Eltern eines Kindes bald auch aus den offiziellen Statistiken verschwinden wird - weil sie zunehmend irrelevant wird und vielleicht doch noch immer zumindest eine Spur von Marginalisierung andeutet. Die absolute rechtliche (kulturelle) Gleichstellung ehelicher und außerehelicher Kinder ist die logische Folge der skizzierten strukturellen Realität, auch wenn latente Marginalisierung als eine - wenn auch inzwischen schnell abnehmende - soziale Sanktionierung der außerehelichen Geburt doch noch hie und da spürbar ist.

Der mit „liberté, „égalité, und „fraternite“, den Leitbegriffen der bürgerlichen Revolution, umrissene Prozeß des Kulturwandels hat - in Interaktion mit einem tiefgehenden Strukturwandel - in einem rund 200 Jahre dauernden Prozeß Zuschreibungen aller Art und daran anknüpfende manifeste Marginalisierungen allmählich weitgehend abgebaut. Das Verschwinden der „Illegitimität“ durch Geburt - und der daraus resultierenden Marginalität - ist ein besonders eindrückliches Indiz für diese fundamentalen Wandlungsprozesse. Die Kirche und das kanonische Recht halten dagegen wandlungsresistent weiterhin an der Zuschreibung von „Illegitimität“ fest und folglich auch an der Dispens.

\section{V.}

Resumieren wir, indem wir abschließend zum Ausgangspunkt zurückkehren. „Illegitimität“ - so hatte ich eingangs gesagt - bezieht sich auf eine "Ordnung“, die eine bestimmte Art von Lebensform als "legitim" definiert und Kinder als „illegitim" bezeichnet und damit marginalisiert, die einer davon abweichenden Beziehung entspringen. Die Institutionen, die solche "Ordnungen“ einführen und durchsetzen konnten, haben im Prozeß eines säkularen und in jüngster Zeit beschleunigten gesamtgesellschaftlichen Wandels sowohl kulturell wie strukturell effektiv an Bedeutung verloren (die Kirche) oder dürfen, können oder wollen ihre Definitions- und Durchsetzungsmacht nicht mehr in einer Weise einsetzen, die bestimmte Personengruppen durch Zuschreibung marginalisiert (der Staat). Staat und weltliches Recht vollziehen damit allerdings nur eine Entwicklung nach, in der ihnen eine individualisierte Gesellschaft mit einigem Vorsprung vorausgegangen ist.

„Legitime Geltung" wird der Heiratsordnung heute - um mit Max Weber ${ }^{27}$ auch zu schließen - weder „kraft Tradition“, „kraft affektuellen ... Glaubens“, „kraft wertrationalen Glaubens" noch „kraft positiver Satzung, an deren Legalität geglaubt wird“, zugeschrieben und kann schon gar nicht „kraft Oktroyierung ... und Fügsamkeit“ durchgesetzt werden. Damit dürfte das Phänomen der „illegitimen“ Kinder - und ihrer Marginalisierung - auch gegen den Widerstand der Kirche wohl endgültig der Vergangenheit angehören. Für Historiker, Soziologen und Juristen bleibt es allerdings ein Forschungsgegenstand, der auch weiterhin noch intensiver Bearbeitung bedarf. 